\title{
Le dire, s'exposer.... ou se taire. Paroles d'enseignant-e-s travaillant sur l'égalité des sexes
}

\section{Gaël Pasquier}

\section{OpenEdition}

\section{Journals}

Édition électronique

URL : http://journals.openedition.org/trema/3574

DOI : $10.4000 /$ trema.3574

ISSN : 2107-0997

Éditeur

Faculté d'Éducation de l'université de Montpellier

Édition imprimée

Date de publication : 1 décembre 2016

Pagination : 14-28

ISBN : 979-10-96627-02-8

ISSN : 1167-315X

Référence électronique

Gaël Pasquier, «Le dire, s'exposer.... ou se taire. Paroles d'enseignant-e-s travaillant sur l'égalité des sexes », Tréma [En ligne], 46 | 2016, mis en ligne le 01 avril 2017, consulté le 19 avril 2019. URL : http:// journals.openedition.org/trema/3574 ; DOI : 10.4000/trema.3574

Ce document a été généré automatiquement le 19 avril 2019.

Trema 


\title{
Le dire, s'exposer.... ou se taire. Paroles d'enseignant-e-s travaillant sur l'égalité des sexes
}

\author{
Gaël Pasquier
}

1 En France, les Instructions Officielles de l'Éducation Nationale préconisent explicitement aux enseignant-e-s de promouvoir l'égalité des filles et des garçons et d'interroger les stéréotypes de sexe depuis les années 80. Dès l'élection de François Mitterrand à la présidence de la République, en 1981, les pouvoirs publics ont cherché, à la suite des mouvements féministes, à faire de l'égalité des sexes à l'école une question politique. Des textes ont ainsi très régulièrement été publiés jusqu'à aujourd'hui, visant des objectifs relativement semblables. Ils restent néanmoins peu connus et jusqu'à une date récente, l'Education nationale ne s'est pas préoccupée de leur application ${ }^{1}$. De fait, ils sont le plus souvent restés lettre morte. En 2013, l'Inspection Générale de l'Éducation nationale a ainsi dénoncé «la faiblesse du pilotage national», un «champ pédagogique encore peu investi ", et le manque de formation initiale et continue des personnels, qualifiant par ailleurs le premier degré «d'angle mort de politiques d'égalité entre filles et garçons » alors même que les stéréotypes de sexe s'y « cristallisent » (IGEN, 2013 pp. 55-56). La prise en compte de ces directives dépend donc en grande partie de la curiosité, du bon vouloir ou du militantisme de certain-e-s enseignant-e-s.

2 Cet article souhaite s'intéresser à ces dernier-e-s et plus précisément à la manière dont les enseignant-e-s d'école maternelle et élémentaire qui mènent dans leur classe un travail en faveur de l'égalité des sexes perçoivent leur situation au sein des équipes? Ces professionnel-le-s sont en effet dans une position paradoxale. Peu nombreux-ses, ils-elles font bien souvent figure d'enseignant-e-s engagé-e-s alors même que leur action se situe dans le cadre de leurs missions : malgré les textes en vigueur, l'égalité des sexes demeure en effet une « question vive » et sa promotion dans le cadre de l'école républicaine ne fait pas consensus (IGEN, 2014). Comment envisagent-ils-elles la particularité de leur situation? Comment décrivent-ils-elles leurs relations professionnelles, entendues au 
sens de pratiques qui structurent formellement ou informellement leurs relations avec leurs collègues au sein de leurs écoles (Lallement, 2008) ? Parlent-ils-elles ou non de cet aspect de leur travail, dans quelle circonstance et quelles réactions celui-ci suscite-t-il ? Comment construisent-ils-elles sa légitimité sur un plan professionnel?

\section{Méthodologie et recueil des données}

3 Cet article est issu d'une recherche visant à mieux connaitre les pratiques des enseignante-s de l'école primaire travaillant avec leurs élèves sur les questions relatives à l'égalité des sexes et des sexualités, pour laquelle vingt entretiens non directifs d'une durée moyenne d'une heure ont été réalisés. L'échantillon des enseignant-e-s rencontré-e-s, plus ou moins informé-e-s des mécanismes de reproduction par l'école de la domination masculine et de l'hétérosexisme, est diversifié sur le plan du sexe (quinze femmes, cinq hommes) $)^{2}$, de l'âge, de l'ancienneté dans la profession (du stagiaire en formation à la conseillère pédagogique récemment retraitée), du niveau d'enseignement (maternelle, élémentaire) et du lieu d'activité (Paris et sa banlieue, des zones urbaines ou rurales de province) (cf. tableau en annexe). Ces personnes ne sont donc pas représentatives du corps enseignant français, mais constituent un ensemble d'informateurs et d'informatrices privilégié-e-s qui témoignent ici des formes que peut prendre une pédagogie qui n'entend pas établir un lien entre le sexe d'un individu et un rôle à jouer dans la société, et des représentations qui la sous-tendent ${ }^{3}$.

4 Les entretiens sont tous antérieurs aux polémiques déclenchées en France par la volonté affichée du ministère de l'Éducation nationale d'expérimenter dans des classes d'école primaire des séances de questionnement contre les stéréotypes de sexe. Ils optaient pour une entrée relativement large : "vous travaillez sur l'égalité des sexes et/ou des sexualités avec vos élèves, je souhaiterais que vous m'en parliez: ce que vous faites, les réactions de vos élèves, des collègues, des parents, ce qui vous semble important". Au cours des entretiens, les personnes rencontrées ont bien souvent été amenées à raconter, parfois longuement, la manière dont elles en étaient venues à aborder ces questions en classe, et évoquer leurs relations avec leurs collègues. Ce sont ces passages que nous analyserons ici. Enfin, nous ne tiendrons pas compte dans cet article des extraits d'entretiens portant sur l'égalité des sexualités car ils soulèvent des questions particulières relatives à la possibilité de parler d'homosexualité à des enfants en bas âge et à l'identification et la visibilité en tant qu'homosexuel-le-s des enseignant-e-s qui le font (Chetcuti , 2010), que, faute de place, nous avons fait le choix de ne pas aborder ici.

\section{Une perception de la réalité et des enjeux sociaux qui isole?}

5 En raison du peu d'intérêt institutionnel manifesté pour l'application des textes officiels de l'Éducation nationale en faveur de l'égalité des filles et des garçons au moment de la réalisation des entretiens (IGEN, 2013), peu d'intérêt relevé à plusieurs reprises dans les entretiens, tenir compte de cette question et l'aborder en classe constitue un choix individuel, propre aux personnes interrogées. Seule une enseignante, Mélanie, a été sollicitée pour intégrer un projet mis en place par la conseillère pédagogique de sa circonscription et ses propos sont l'exception qui vient confirmer notre affirmation 
puisqu'elle annonce un an après cette expérience avoir mis entre parenthèses la question de l'égalité des sexes dans sa classe ne la jugeant pas prioritaire. Travailler en faveur de l'égalité des sexes implique donc de s'intéresser à une question à laquelle la plupart des collègues tendent à opposer une certaine indifférence :

"toutes les autres [collègues] et encore plus celles de l'ancienne école [cette enseignante a changé d'école], pff ça leur passe complètement à côté; alors je leur donne mes bibliographies, je dis "mais prenez, mais regardez, il y a des ouvrages qui ont été écrits dessus; mais lisez, documentez-vous et vous allez voir que ce n'est pas si simple que ça" " (Julie).

6 Les entretiens mettent en évidence que l'adoption d'une perspective qui entend déconstruire le système de genre, c'est-à-dire un «système [bicatégoriel] de normes de sexe hiérarchisant, producteur d'inégalités, qui légitime ces inégalités en les naturalisant [sous les habits de LA différence des sexes] ( (Marro, 2012: 68), ou pour reprendre une expression imagée courante, l'observation des phénomènes éducatifs et des relations professionnelles à travers "les lunettes du genre» entraine souvent une impression d'isolement. Ce mode de lecture du monde, qu'une enseignante compare à un engrenage et qu'il semble impossible d'abandonner une fois effectuée une première prise de conscience des inégalités, pose donc en permanence, la question du positionnement à adopter face à certaines situations car il est loin d'être partagé. La prise de parole pour parler de son travail en faveur de l'égalité des sexes, relever ou contester une situation ou certains propos, n'est ainsi pas sans conséquences. Elle porte en elle un potentiel conflictuel dont l'individu doit mesurer l'impact. Certaines enseignant-e-s rencontré-e-s choisissent ainsi de ne pas faire part de leurs convictions, ni de leur action avec leurs collègues afin de conserver une certaine sérénité dans l'équipe enseignante :

"avec les collègues ça va; l'une [d'entre elles] surtout est très hermétique; on pense différemment et impossible d'en parler donc bon, je me bats pas non plus, je suis pas militante quand même à ce point (rire); enfin je pense pas qu'il faille imposer de manière prosélyte des idées parce que faut laisser les gens...; moi quand j'ai abordé le thème en sciences de l'éducation justement sur les discriminations, j'étais comme tout le monde: d'abord, en premier lieu, j'ai dit "bon qu'est-ce qu'on me raconte?; on [les hommes et les femmes] est bien différent..." ; et puis, ça se travaille, ça se réfléchit, ça s'analyse et c'est à force qu'on découvre comment les préjugés se construisent " (Béatrice).

7 Alors qu'elle analyse «le processus de création de contextes propices à la discussion politique » dans le cadre de son analyse des stratégies d'évitement des questions qui en relèvent dans les conversations quotidiennes des «Américains ", Nina Eliasoph reprend un terme utilisé par Erving Goffman qui nous semble particulièrement bien convenir aux situations des enseignant-e-s qui nous ont été racontées : «Goffman a appelé footing ce processus tacite incessant par lequel on vérifie où l'on met les pieds avant chaque interaction (Goffman, 1979) est-ce qu'il y a des escaliers? des gravillons? De la glace? Pour avancer, il faut tâter le terrain.

$8 \quad$ Il en est de même de la prise de parole : est-ce qu'on parle pour faire la conversation? Pour accomplir une tâche? Pour se mettre en avant? Pour donner sens aux interactions, nous puisons dans ce réservoir inépuisable de "savoirs communs" qui permet aux participants d'interpréter mutuellement leurs propos » (Eliasoph, 1998, pp. 33-34).

9 Faire part de son désaccord et de ses convictions dans un cadre professionnel, notamment sur des questions d'ordre professionnelles, la reproduction par l'école des inégalités entre les sexes, implique donc de savoir où l'on met les pieds car cela n'est potentiellement pas sans conséquences. Un tel positionnement implique de s'exposer à son tour à des formes 
d'hostilité, à la possible désapprobation de ses collègues ou des autres personnes présentes, d'autant que les situations mettant en jeu l'égalité des sexes sont fréquentes, voire permanentes. C'est donc prendre le risque de se marginaliser dans un cadre familier. Certain-e-s enseignant-e-s préfèrent donc user d'une certaine prudence en évaluant les risques encourus à l'aune de ce qui est dit, des personnes qui le disent, mais également, à l'instar de Béatrice, en référence à la temporalité de leur propre cheminement sur ces questions. Se forment ainsi petit à petit dans certaines écoles des «bulles » affinitaires où l'on sait pouvoir parler de certaines choses. Mais l'instauration de cette complicité ne rend pas moins compliqué d'avoir à évoquer ces sujets hors de ce cadre protecteur, notamment dans un contexte institutionnel qui réunit l'ensemble des collègues de l'école: "bon, là je parle des discussions informelles quand on surveille la récréation, par contre c'est des sujets qu'on n'aborde pas du tout en conseil de maîtres ou en conseil de Cycles» (Sophie).

D'autres enseignantes, au contraire, choisissent d'intervenir, justement pour activer une prise de conscience :

" les instits comme le reste de la société se traînent aussi avec leurs stéréotypes et d'entendre un collègue répondre à un élève qui lui demandait qu'on lui attache ses lacets, "tiens demande à une fille, faut bien que ça serve", bon moi évidemment je réagis: "mais enfin tu te rends compte de ce que tu dis à un petit garçon de six ans, de l'impact"; je crois que les enseignants n'ont pas non plus conscience de la valeur de leur parole face à des enfants et que ce n'est pas sérieux quand l'adulte se positionne et dit des choses comme ça» (Mireille).

11 Par son intervention, Mireille rompt avec le ton en apparence badin de son collègue. Elle oppose ainsi une lecture en termes de domination, de respect, de responsabilité éducative, à ce que son collègue regardait sans doute comme un simple trait d'humour. Ses propos invitent donc à penser l'expression d'une citoyenneté dans un cadre professionnel et les stratégies mises en œuvre par des individus pour défendre des valeurs théoriquement conformes à l'idéal démocratique d'égalité mais dont la pertinence n'est pas toujours reconnue au-delà d'un consensus de façade. Les situations racontées par les personnes rencontrées mettent en effet bien souvent en jeu deux lectures inconciliables du monde : «ce qui est très difficile, c'est de ne pas être compris, c'est-àdire qu'une fois qu'on est rentré dans l'analyse politique de la chose on se comprend [mais] la personne en face ne comprend pas ce qu'on veut dire... ( (Mireille). L'une est politique et entend contester des rapports de pouvoirs entre groupes sociaux à l'œuvre dans des situations en apparence banales et quotidiennes, tandis que l'autre les analyses en termes de relations mettant en jeu des individus par principe libres et égaux, et préfere considérer certains choix comme le résultat de décisions personnelles qui doivent être respectées plutôt que d'y voir l'expression d'une forme de domination: «alors c'est vrai que y'en a qui vivent [l'inégalité] au quotidien et qui la ressentent pas comme une inégalité " (Danièle). Ce dernier point de vue invisibilise les rapports de force à l'œuvre dans la société pour leur substituer des préférences individuelles, souvent naturalisées pour le sujet qui nous préoccupe, par l'affirmation de différences incommensurables entre les sexes.

En fonction des contextes les personnes rencontrées vont donc choisir de faire part ou non de leurs opinions. Une prise de position pourra sembler à certains moments superflue et inappropriée, entrainant ce que Nina Eliasoph qui reprend l'expression à Elizabeth Noelle-Neuman appelle " spirale du silence ». " Elle décrit ainsi le processus par lequel les gens - qui sont d'après elle, par nature désireux de bien s'entendre - 
s'abstiennent d'exprimer des points de vue qu'ils estiment non conventionnels devant des inconnus » (Eliasoph, 1998, p. 63). Bien qu'il soit possible de contester, comme le fait la sociologue, la pertinence de la catégorie d' « inconnu », qui n'est pas toujours adéquate pour rendre compte des interactions décrites dans nos entretiens, c'est donc bien l'opportunité de faire entendre un avis dissonant et risquant de rendre la suite de la conversation plus tendue qui est questionnée en situation par certain-e-s enseignant-e-s rencontré-e-s et peut entraîner parfois des réponses diverses. Dans d'autres occasions, il semblera en effet impossible à certaines personnes de ne pas réagir, notamment, comme dans la situation rapportée par Mireille, dans un contexte éducatif où la parole des adultes est dotée de l'autorité du savoir et de la fonction qu'ils-elles occupent.

Il ne s'agit pas ici de déterminer des règles qui expliqueraient pourquoi tel sujet est abordé dans tel contexte et non dans tel autre. En revanche, il est intéressant de constater que ces propos témoignent des négociations et stratégies permanentes mises en œuvre par les personnes interrogées pour évaluer s'il est approprié ou non d'aborder la question de l'égalité des sexes dans une situation donnée, et ce alors même qu'elles précisent à d'autres moments s'en préoccuper tous les jours dans leur classe et être continuellement ou tout au moins très régulièrement confrontées dans leur école à des paroles, des attitudes ou des comportement qui manifestent de façon plus ou moins explicite la domination des hommes sur les femmes ${ }^{4}$.

Par ailleurs, plusieurs entretiens montrent que cette appartenance des personnes qui travaillent sur l'égalité des sexes à la catégorie "femmes " peut être perçue comme un moyen de dévaluer leur parole et leur action : à travers leur implication vis-à-vis d'un sujet qui nécessite en grande partie de rendre les femmes visibles et de leur donner accès à des domaines qui leur étaient refusés, c'est leur manque de neutralité présumé qui est souligné. Dès lors, pour que leurs idées soient dans ces situations considérées comme recevables, il faudrait qu'elles soient également portées par les hommes ${ }^{5}$, ce qui reviendrait alors à demander l'assentiment des dominants à la mise à bas de leur domination. Ainsi, alors qu'elle prévient sa directrice qu'elle compte aborder la question des stéréotypes de sexe dans sa classe, Hélène raconte que celle-ci lui laisse entendre que sa position est intrinsèquement biaisée : «elle m'a dit "oui, mais il faudrait en parler au prof de sport parce que c'est pas recevable si vous parlez de ça et si y'a qu'une femme qui parle de ça" " (Hélène). Bien qu'il ne soit pas possible de savoir si la réponse de la directrice d'Hélène aurait été la même si cette demande lui avait été faite par un homme, elle indique dans ce cas précis que pour elle, les avis d'une femme sur la question des stéréotypes de sexe sont forcément partiaux, suspects, et qu'il importe de leur adjoindre ceux du professeur de sport, seul homme de l'équipe, afin de les contrebalancer, comme si, en raison de leur différence de sexe ceux-ci allaient être "naturellement» complémentaires. Les recherches féministes en général que ce soit en histoire, en éducation, en sociologie, ont fait depuis longtemps face à la critique de la partialité, et ont été perçues comme des recherches militantes et non objectives... La critique féministe a quant à elle questionné le paradigme classique de la neutralité scientifique s'appuyant sur les représentations d'hommes blancs hétérosexuels (Dorlin 2008, pp. 28-30 ; Hill Collins, 1989, pp. 142-147). 


\section{La visibilité, source de nouveaux questionnements, de nouvelles représentations mais aussi de nouvelles solidarités?} lorsqu'elles abordent des thématiques qui font pourtant partie des missions revendiquées par l'institution dont ils-elles sont les agent-e-s. Les entretiens montrent que ce sentiment de solitude très largement partagé doit être nuancé. Il s'agit avant tout d'une solitude dans le quotidien de leur travail, car presque toutes et tous les enseignant-e-s interrogé-e-s ont rencontré au cours de leur carrière des personnes préoccupées par ces questions qui leur ont donné des pistes de travail, les ont encouragés dans cette direction et permis de construire la légitimité professionnelle de leur engagement pédagogique. Ce peut être un-e professeur-e d'IUFM ${ }^{6}$, plus ou moins informé-e de ces questions mais qui accepte d'encadrer un mémoire professionnel que d'autres de ses collègues ont parfois refusé ; ou encore qui intègre cette dimension dans ses cours. Ce peut être une amie qui travaille dans le domaine de l'enseignement et qui indique des documents pédagogiques disponibles pour travailler sur l'égalité des sexes; un conseiller pédagogique particulièrement investi sur ces questions; ou encore une inspectrice ou un inspecteur qui soutient des initiatives à l'échelle de sa circonscription parce qu'elle-il est persuadé-e de leur bien-fondé. Petit à petit, les enseignant-e-s rencontré-e-s se construisent donc un réseau qui leur permet parfois d'intégrer des groupes de travail internes ou externes à l'Éducation nationale. Mais ces rencontres peuvent leur donner l'impression d'être trop limitées et sont peu représentatives de ce qui se passe au jour le jour dans les écoles. Il ressort par ailleurs de ces récits, à l'instar de ce que constate l'IGEN (2013), que ce n'est que très rarement l'institution en tant que telle qui promeut l'égalité des sexes à l'école mais toujours des individu-e-s particulier-e-s qui prennent appui sur leur fonction et leurs missions pour défendre une certaine conception de l'éducation. Ce sont des initiatives individuelles, qui parfois arrivent à se relier entre elles malgré l'éloignement géographique (Sandrine et Danièle) mais qui peinent souvent à essaimer au-delà du cercle des personnes déjà convaincues du bien-fondé de leur travail.

Par ailleurs, alors que certains enseignant-e-s disent aborder les questions d'égalité des sexes et parfois d'égalité des sexualités dans les conseils des maîtres-ses de leur école (Delphine, Nathalie, Éric), de telles initiatives ne semblent pas pour autant aisées. Delphine qualifie ainsi de " putsch » le fait d'avoir réussi à inscrire avec l'aide de l'une de ses collègues le refus des discriminations sexistes et homophobes dans le règlement intérieur de son établissement et constate quatre ans après le peu de traduction dans les faits de cette affirmation de principe. Tout au plus lui a-t-elle permis de renforcer la légitimité de sa propre action aux yeux de l'ensemble des élèves. D'autres enseignantes remarquent ne pas réussir à impulser une dynamique d'action commune pour promouvoir une autre organisation d'espaces de l'école, comme la cour de récréation, alors même que leurs collègues reconnaissent qu'ils reposent sur un ordre inégalitaire (Pasquier, 2015).

Malgré ces constats parfois pessimistes et même si les personnes rencontré-e-s associent parfois le fait d'être identifié-e-s comme féministes à une prise de risques qui peut les mettre en danger professionnellement, les entretiens montrent que la présence dans les écoles de personnes perçues comme soucieuses de l'égalité des sexes permet l'apparition 
de poches de résistance à la domination masculine où il est possible pour les collègues, les parents mais aussi les enfants de penser des questions jusque-là invisibles et de s'autoriser eux-elles aussi à certaines formes de visibilité. Ces situations de reconnaissance peuvent par exemple amener certain-e-s enseignant-e-s de l'équipe, également sensibles à la question, à souhaiter engager un travail commun lors de la rentrée suivante :

"mes collègues sont plus réfractaires; une seule voudrait qu'on bosse mon projet ensemble l'année prochaine; elle voit le sens de ce que je fais en classe et pourquoi je suis si vigilante aux rapports garçons filles c'est la seule; peut-être parce que à l'IUFM elle a été amenée à avoir une conférence sur ça, donc elle a été alertée » (Julie).

D'autres réactions, plus ambigües, si elles ne touchent pas les questions professionnelles, n'en participent pas moins à la mise en place de relations de solidarité :

" alors, par contre, si jamais y'en a une [collègue] qui se fait avorter, je serai la première à le savoir ; elle va venir m'en parler discrètement entre deux portes, si elle a des problèmes avec son mari et qu'elle veut un numéro SOS Femmes battues; j'ai eu le cas: dès qu'on est reconnue féministe, on est reconnue comme confidente; c'est à moi qu'elles vont en parler et surtout pas en salle des maîtres; et quand on est en groupe par contre, ces échanges un peu privés qu'on a eu, du domaine personnel, très personnel, la sexualité tout ça, et ben d'un seul coup elles s'éloignent de moi, elles sont à l'autre bout [de la pièce] et surtout "je lui parle pas" ; enfin moi je ressens ça comme ça, surtout quand on arrive dans une nouvelle école » (Mireille).

19 Le comportement des collègues de Mireille qui oscille entre des marques de confiance et des stratégies d'évitement afin de ne pas porter le "stigmate"7 de son féminisme revendiqué est proche d'une situation rapportée par Didier Eribon dans ses Réflexions sur la question gay au cours de laquelle André Gide, s'assoit à la table d'Oscar Wilde de manière à tourner le dos au passant (Eribon 1999, pp. 76-77). Dans les deux cas, ce n'est pas la proximité physique avec une personne "stigmatisée » qui est redoutée, mais cette proximité physique sous les yeux des autres, ces derniers agissant alors comme un ferment contagieux propre à discréditer celui ou celle qui ne l'était pas encore. Pour autant, ces relations sont aussi, d'une certaine manière, des relations de solidarité puisque les collègues de Mireille savent qu'elles peuvent trouver auprès d'elle une aide, des informations ou tout simplement une écoute bienveillante. Mireille leur permet en effet de rompre un isolement et d'ouvrir une réflexion à propos de sujets intimes ou de souffrances toujours singulières qui participent de l'expérience collective des femmes en tant que groupe discriminé. Ces moments d'échanges entre deux portes peuvent également constituer une étape vers une prise de conscience plus importante qui permettra ensuite de montrer cette intimité sans l'associer à un sentiment de honte. À travers ces relations dans un cadre professionnel, motivées par des préoccupations qui ne le sont pas, se nouent en effet des possibilités d'émancipation par rapport aux contraintes du système de genre.

Vis-à-vis des élèves, cette identification en tant qu'enseignant-e soucieux-se d'égalité des sexes donne également aux enfants la possibilité de formuler des plaintes sur des questions qui ne suscitent habituellement pas l'attention de l'équipe enseignante, de demander des informations sur des sujets qu'ils-elles n'oseraient pas nécessairement aborder avec d'autres adultes ou simplement de faire part de leur ressenti vis-à-vis des questions abordées en classe. De son côté, Camille qui organise des temps de boxe nonmixtes destinés aux filles afin de les aider à s'affirmer et leur permettre d'acquérir un répertoire moteur auquel elles n'ont habituellement pas accès remarque que son initiative est reçue avec enthousiasme : « là, je suis arrivée dans l'école, toute les filles me 
disaient "tu me prends l'année prochaine à ton atelier ?" ; enfin y'a la queue, je te dis pas » (Camille). Dans le quotidien des écoles, les pratiques des enseignant-e-s rencontrée-s ouvrent donc un répertoire de comportements et d'attitudes possibles et dicibles qui n'existait pas avant, et permettent de penser des réalités jusque-là ignorées.

\section{Discussion conclusive}

21 À partir d'entretiens réalisés avec des professeur-e-s des écoles travaillant avec leurs élèves sur les questions d'égalité des sexes et des sexualités, cet article souhaitait rendre compte de ce qui se joue dans les relations professionnelles entre collègues autour de ces questions. De fait, les propos des personnes rencontrées illustrent et permettent de contextualiser la situation paradoxale que nous avons identifiée en introduction. Malgré l'existence de politiques éducatives en faveur de l'égalité des sexes, les enseignant-e-s interrogé-e-s disent mener des actions relativement solitaires et s'interroger en permanence sur leur positionnement professionnel, notamment vis-à-vis de leurs collègues. Parler de son action, réagir en situation n'est en effet pas toujours sans conséquence et peut être renvoyé à un engagement féministe et personnel dont la légitimité dans un cadre scolaire serait sujette à caution. De ce point de vue, le matériel recueilli ne permet pas de déterminer si la situation des femmes est particulière par rapport à celle des hommes, bien qu'il soit probable que la perception de leur implication dans le combat pour l'égalité des sexes rende leur position plus inconfortable. Sur les cinq hommes interrogés, quatre se définissent en effet comme homosexuels: c'est donc davantage autour de la question de la visibilité de leur orientation sexuelle que se pose la question de leur engagement au cours des entretiens, celui-ci portant à chaque fois sur l'égalité des sexualités à laquelle est souvent associée l'égalité des sexes. Le cinquième qui précise juste au cours de l'entretien avoir des enfants, sans donner d'indication sur une possible orientation sexuelle, mentionne uniquement une équipe enseignante solidaire dans un contexte d'enseignement difficile, ce qui validerait notre hypothèse, selon laquelle l'engagement des hommes en faveur de l'égalité des sexes est perçu comme moins problématique que celui des femmes.

Il peut en outre sembler étonnant que les textes officiels de l'Éducation nationale en faveur de l'égalité comme la Convention interministérielle pour l'égalité des chances entre les filles et les garçons, les femmes et les hommes dans le système éducation en vigueur au moment de la réalisation des entretiens ne soient pas davantage sollicités par les personnes rencontrées. Ces textes ne sont en effet jamais mentionnés pour tenter de construire un travail en équipe en lien avec les missions de l'école. En revanche, ils le sont en général lorsque se manifeste la crainte d'avoir à justifier son action en faveur de l'égalité des sexes et des sexualités à des interlocuteur-rice-s peu convaincu-e-s de son bien-fondé, bien souvent un parent d'élèves et joue de ce fait essentiellement un rôle protecteur. Il est possible de voir dans cette attitude une forme de soupçon vis-à-vis d'injonctions venant d'en haut dans une institution qui tend à les multiplier sans se soucier des possibilités d'opérationnalisation effective. En même temps qu'il met en évidence l'absence de consensus sur les questions d'égalité des sexes à l'école, y compris au sein d'un même corps professionnel, un tel positionnement illustre également le manque de volonté de l'institution à faire appliquer ces textes ${ }^{8}$, au point que les professionnel-le-s les envisagent uniquement comme des recours a postériori. Certains enseignant-e-s rencontré-e-s déplorent ainsi que les programmes du premier degré qui constituent à 
l'inverse des Conventions et des circulaires des textes contraignants ne fassent pas plus de cas d'égalité des sexes. C'est donc bien l'absence de réelle démarche politique et institutionnelle que ces usages révèlent.

De fait, les enseignant-e-s interrogé-e-s semblent se laisser enfermer dans une vision stéréotypée et militante dont ils-elles peinent à se dégager. Qu'ils-elles revendiquent ou non cette dernière, travailler sur l'égalité des sexes s'inscrit en effet dans une tentative d'articulation d'une préoccupation personnelle avec une perspective pédagogique. Certain-e-s enseignant-e-s rencontré-e-s notent ainsi qu'il n'est pas possible de démêler ces différents éléments dans leurs pratiques ou celles de leurs collègues, tant ils sont à leurs yeux intrinsèquement liés, notamment parce que les questions relatives à l'égalité des sexes interrogent profondément les façons d'enseigner (Pasquier, 2013, 2016). Il n'est donc pas anodin, que, dans le cadre d'un entretien sur leur travail en classe sur ces questions, certain-e-s d'entre elles et eux prennent la peine de raconter leur prise de conscience des inégalités et de retracer un itinéraire militant qui aboutit à la prise en compte de ces questions dans leur classe. Le travail réalisé avec les élèves autour de l'égalité des sexes est donc pensé en référence à ces engagements qui le nourrissent et qu'il interpelle en retour.

24 En conséquence, l'image militante et engagée ou simplement impliquée à laquelle ces enseignant-e-s sont renvoyées par leurs collègues est précisément celle à partir de laquelle ils-elles ont bien souvent construit pour eux-elles le sens de leur action, ce qui explique également le caractère occasionnel des références aux textes officiels dans les entretiens.

Les sphères personnelles et professionnelles sont donc à envisager en termes d'interdépendance et ne peuvent être analysées comme des univers qu'il serait possible de dissocier (Cacouault-Bitaud, 2007), perspective d'analyse qui permet tout autant de comprendre et saisir l'engagement des enseignant-e-s rencontrées que la force de certaines résistances aux questions de genre en éducation. De fait, la confrontation de nos entretiens avec certains récits de formations d'enseignant-e-s à la question du genre en éducation, laisse apparaitre un paradoxe : alors que certain-e-s formateur-rice-s, disent travailler avec leurs stagiaires sur la dissociation entre ce qui peut dépendre de choix privés et ce qui relève des obligations des fonctionnaires de l'État (Baurens et Schreiber, 2010), les enseignant-e-s que nous avons rencontré-e-s construisent leur action à partir d'enjeux personnels forts et ne recourent qu'occasionnellement aux instructions officielles pour la légitimer.

La mise en œuvre des politiques éducatives en faveur de l'égalité des sexes et des sexualités réaffirme donc en même temps qu'elle la questionne la pertinence de la dichotomie vie publique / vie privée telle que la conçoit la théorie libérale classique et telle que l'a mise en cause la critique féministe en sciences politique et sociale (Forquin, 2003 ; Moller Okin, 1991). Loin de dénier le droit de chacune et chacun à la vie privée et à l'intimité, cette dernière a montré, notamment à travers le slogan «le personnel est politique ", "que ce qui se passe dans la vie personnelle, en particulier dans les relations entre les sexes, n'est pas imperméable à la dynamique du pouvoir, qui est généralement caractéristique du politique »; en conséquence, "ni le domaine de la vie domestique et personnelle, ni celui de la vie non domestique, économique et politique, ne peuvent être compris ou interprétés isolément l'un de l'autre » (Moller Okin, 1991, p. 363). La prise en compte des questions relatives à l'égalité des sexes au sein de la classe interroge ainsi les représentations que se font les enseignant-e-s de ce qu'est un homme ou une femme mais 
aussi la manière dont chacune et chacun a construit son identité professionnelle dans un métier majoritairement exercé par des femmes. En même temps qu'elles testent la possibilité d'une action pédagogique qui permettrait de rompre avec les phénomènes de reproduction sociale, les pratiques des enseignant-e-s rencontré-e-s interfèrent avec leur questionnement identitaire et leur itinéraire de vie. C'est cette porosité à l'œuvre dans le travail en faveur de l'égalité des sexes et des sexualités à l'école, la manière dont elle l'informe dans une relation réciproque et dynamique, qu'il serait intéressant d'interroger plus avant.

Annexe : Tableau récapitulatif des entretiens

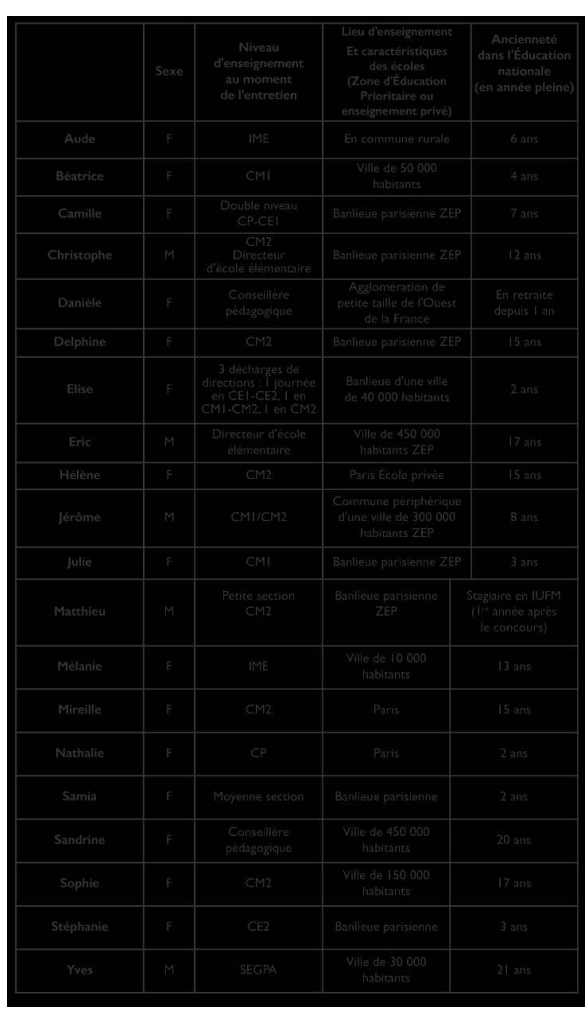

\section{BIBLIOGRAPHIE}

Bard, C. (2012). Le féminisme au-delà des idées reçues. Paris : Le cavalier bleu éditions.

Baurens, M. et Schreiber, C. (2010). Comment troubler les jeunes enseignant-e-s sur la question du genre à l'école? Analyse d'une expérience de six ans de formation en IUFM. Nouvelles Questions Féministes vol. 29 (2), 72-86.

Buscatto, M., Chevalier, Y., Collet, I. et al. (2015). Égalité des sexes à l'école : machine arrière, toute ! Médiapart, 16 janvier 2015. http://blogs.mediapart.fr/edition/les-batailles-de-legalite/ article/160115/egalite-des-sexes-1-ecole-machine-arriere-toute [consulté le 10/05/2015]. 
Cacouault-Bitaud, M. (2007). Professeurs... mais femmes. Carrières et vies privées des enseignantes du secondaire au XXe siècle. Paris : La découverte.

Chetcuti, N. (2010). Se dire lesbienne. Vie de couple, sexualité, représentation de soi. Paris : Payot.

Delcroix, C. (2009). Professeur-e-s des écoles : carrières et promotions. Les identités professionnelles sexuées des enseignant-e-s du premier degré. Thèse de Doctorat. Université Paris X - Ouest Nanterre La Défense.

Dorlin, E. (2008). Sexe, genre et sexualités. Paris : PUF.

Draelants, H. (2009). Réforme pédagogique et légitimation. Le cas d'une politique de lutte contre le redoublement. Bruxelles : De Boeck.

Eribon, D. (1999). Réflexions sur la question gay. Paris : Fayard.

Eliasoph, N. (1998/2010). L'évitement du politique. Comment les Américains produisent l'apathie dans la vie quotidienne. Paris : Economica.

Forquin, J.-C. (2003). La critique communautarienne du libéralisme politique et ses implications possibles pour l'éducation. Revue Française de Pédagogie n¹43, 113-139.

Goffman, E. (trad. 1975). Stigmate. Les usages sociaux des handicaps. Paris : Éditions de minuit.

Goffman, E. (trad. 1987). Façons de parler. Paris : Éditions de Minuit

Hill Collins, P. (1989/2008). La construction sociale de la pensée féministe Noire., In E. Dorlin (dir.), Black féminism. Anthologie du féminisme africain-américain, 1975-2000 (pp. 135-175). Paris : L'Harmattan.

Inspection générale de l'Éducation nationale (IGEN) (2013). L'égalité entre filles et garçons dans les écoles et les établissements. Paris : ministère de l'Éducation nationale.

IGEN (2014). Évaluation du dispositif expérimental « ABCD de l'égalité ». Paris : MEN.

INSEE, 2016. Enseignants du public et du privé par corps en 2015, janvier 2016, http:// www.insee.fr/fr/themes/tableau.asp?reg_id=0\&ref_id=nattef07115 [consulté le 10/05/2016].

Lallement, M. (2008). Sociologie des relations professionnelles. Paris : La Découverte.

Löwy, I. et Marry, C. (2007). Pour en finir avec la domination masculine. De A ̀̀ Z. Paris : Les empêcheurs de penser en rond.

Marro, C. (2012). Dépendance-indépendance à l'égard du genre. Penser l'égalité des sexes au-delà de LA différence. Recherche et Formation 69, 65-80.

Ministère de l'Éducation nationale (2006). Convention interministérielle pour l'égalité des chances entre les filles et les garçons, les femmes et les hommes dans le système éducation. Paris : MEN.

Moller Okin, S. (1991/2000). Le genre, le public et le privé. In T.-H., Ballmer-Cao ; V. Mottier et L. SGIER Lea (dir.), Genre et politique. Débats et perspectives (pp. 345-396). Paris : Gallimard.

Pasquier, G. (2013). L'enseignement de la langue française à l'école primaire à l'épreuve de la déconstruction du genre. Formation et pratiques d'enseignement en questions $\mathrm{n}^{\circ} 16,161-178$

Pasquier, G. (2015). La cour de récréation au prisme du genre, lieu de transformation des responsabilités des enseignant-e-s à l'école primaire. Revue des Sciences de l'éducation $\mathrm{n}^{\circ} 41$ (1), 91-114.

Pasquier, G. (2016 à paraître). Du contrôle de ses actions à l'implication des élèves : la mise en place d'une gestion égalitaire de la prise de parole entre les filles et les garçons par des 
enseignant-e-s d'école primaire. In M. Baurens, A. Lechenet et I. Collet (dir.), Former à l'égalité : défi pour une mixité véritable. Paris, L'Harmattan.

Pasquier, G. et Passerieux, C. (2015). Du projet de programme de l'école maternelle à sa version définitive, des choix problématiques pour construire l'égalité ?, Intervention lors de la journée d'étude Les nouveaux programmes de l'école maternelle. Quels enjeux à la lumière des recherches récentes sur la petite enfance. École Supérieure du Professorat et de l'Éducation de l'académie de Créteil, 30 septembre 2015. Site du Groupe Français d'Éducation Nouvelle (GFEN), http://www.gfen.asso.fr/ images/documents/dossier_sans_titre/passerieux_pasquier_pr_maternelle_analyses_2015.pdf [consulté le 11/12/2015].

Petrovic, C. (2010). Partage d'expérience sur les formations "genre et éducation" et évolutions ». In C. Morin-Messabel (dir.), Filles/Garçons. Questions de genre, de la formation à l'enseignement (pp. 187-202). Lyon : Presses Universitaires de Lyon.

Thiers-vidal, I. (2010). De "L'ennemi Principal" aux principaux ennemis. Position vécue, subjectivité et conscience masculines de dominations. Paris : L'Harmattan.

\section{NOTES}

1. Il est encore trop tôt pour savoir si le programme $A B C D$ de l'égalité mis en œuvre conjointement par le ministère de l'Education Nationale et le ministère des Droits des femmes durant l'année scolaire 2012-2013 a été suivi d'effets, d'autant que le gouvernement y a mis un terme dès la rentrée suivante. Le plan d'action qui l'a remplacé en 2014 semble en effet plus reposer sur des effets d'annonce que sur une volonté politique réelle de faire appliquer les directives existantes, d'autant que les textes actuellement publiés par l'institution ne manquent pas d'être problématiques et/ou ambigus quant à la manière dont l'Éducation nationale conçoit et entend promouvoir l'égalité des sexes (Buscatto, Chevalier, Collet et al. 2015 ; Pasquier et Passerieux 2015).

2. Les hommes y sont donc légèrement sur-représentés puisque les enseignant-e-s du premier degré (maternelle et élémentaire), comptent 82,6 \% de femmes (INSEE, 2016).

3. Pour une description détaillée de la méthode, voir Pasquier, 2015.

4. La revendication de l'appartenance au mouvement féministe ou sa mise à distance dans un contexte professionnel ou privé, ne peut donc pas uniquement être lue comme un signe d'adhésion ou de rejet des valeurs portées par celui-ci. Elle doit être remise en contexte en fonction des risques auxquels la personne qui parle estime s'exposer à s'en réclamer, compte tenu des représentations dépréciatives dont elle est fréquemment l'objet (Bard, 2012). Les stratégies de résistance et d'agression verbale déguisées en plaisanteries auxquelles peuvent être confrontées certaines enseignantes rencontrées visent clairement à délégitimer leurs convictions. L'objectif est de faire passer ces dernières comme anachroniques, déconnectées de la réalité, alors même que tout dans ces réactions témoigne de la prégnance du système de genre. Les remarques faites sur l'absence d'humour des féministes ou les problèmes conjugaux supposés des femmes qui se préoccuperaient d'égalité des sexes visent à les transformer en problèmes d'ordre personnel, qui exprimeraient avant tout leur inadaptation sociale mais aussi à les renvoyer de manière détournée à la catégorie dominée à laquelle elles appartiennent : l'hystérie supposée des féministes doit en effet être lue comme l'expression d'une des composantes de la « nature » des femmes en général (Löwy et Marry, 2007).

5. Bien que l'engagement des hommes dans les combats des femmes n'est pas nouveau, il reste bien souvent le fait d'une minorité et dont les motivations sont parfois ambivalentes (ThiersVidal, 2010). 
6. Institut Universitaire de Formation des Maîtres, aujourd'hui remplacés par les Écoles Supérieures du Professorat et de l'Éducation (ESPE).

7. «Le mot de stigmate servira [...] à désigner un attribut qui jette un discrédit profond, mais il faut bien voir qu'en réalité c'est en termes de relations et non d'attributs qu'il convient de parler» (Goffman 1975, p.13).

8. Pour une analyse similaire à partir des politiques éducatives concernant le redoublement, $c f$. Draelants, 2009.

\section{RÉSUMÉS}

Cet article porte sur une situation particulière : celle des enseignant-e-s d'école primaire qui prennent en compte dans leur classe la question de l'égalité des sexes. Il s'intéresse plus précisément à la manière dont ces dernier-ère-s perçoivent leur situation au sein des équipes enseignantes. Ces professionnel-le-s sont en effet dans une position paradoxale. Peu nombreux, ils-elles font bien souvent figure d'enseignant-e-s engagés alors même que leur action se situe dans le cadre de leurs missions.

This article deals with the particular situation of the teachers who take into account in their classroom the question of sex equality. More precisely he focuses on the way those teachers perceive their situation in the teaching teams. Those professionnals are indeed in a paradoxal position. They are not nu-merous and they are perceived very often like politically engaged teachers even though their action is comprised in the limits of their missions.

\section{INDEX}

Mots-clés : genre, égalité des sexes, école maternelle et élémentaire, pratiques enseignantes, professionnalité enseignante

Keywords : gender, sex equality, Primary school, teaching practices, teaching professionality

\section{AUTEUR}

\section{GAËL PASQUIER}

Maître de conférences en sociologie, ESPE - Université Paris Est Créteil (UPEC), Observatoire Universitaire International Éducation et Prévention (OUIEP), Laboratoire Interdisciplinaire de Recherche sur les Transformations des pratiques Educatives et des pratiques Sociales (LIRTES) 